\title{
Updated classification and novel treatment prospective for nodal peripheral T-cell lymphomas
}

\author{
“...although it has been shown to be potentially \\ curative for approximately $40 \%$ of patients affected by \\ relapsed/refractory PTCL, at present there is no \\ evidence that allogeneic stem cell transplantation is \\ superior to autologous stem cell transplantation for \\ patients in first remission after induction therapy."
}

\section{Pier Paolo Piccaluga ${ }^{* 1,2}$}

First draft submitted: 9 March 2017; Accepted for publication: 28 March 2017; Published online: 30 June 2017

Peripheral T-cell lymphomas (PTCLs) are uncommon diseases representing $10-15 \%$ of all lymphomas. They are defined as rare in Europe and the USA, while they are more frequent in Asia as well as in the Caribbean area [1]. Recently, an international study led by Massimo Federico enrolled 1510 cases from 75 centers worldwide, updating some epidemiological and clinical information [2]. The most frequent subtype turned out to be PTCL not otherwise specified (NOS) (36\%). Distribution of different subtypes among geographic areas corresponded to previous data, confirming that angioimmunoblastic lymphoma (AITL) is more frequent in Europe and the USA (21\% each), anaplastic large cell lymphoma (ALCL) anaplastic lymphoma kinase (ALK)-negative in South America (26\%) and natural killer (NK)/T-cell lymphoma (NKTCL) in Asia (30\%). Data on therapy were available for 1022 patients: chemotherapy plus/minus radiotherapy was the preferred induction approach. Transplants were carried out in $20 \%$ of patients, with some different habits among areas (USA 13\%, EU 8\%, Asia 6\% and South America 2\%). PTCLs were confirmed to be aggressive tumors; after a median follow-up of 39 months, 605 deaths have been recorded (43\%), $71 \%$ of them from lymphoma; the 5-year overall survival (OS) and 5-year progression-free survival (PFS) were 42 and $32 \%$, respectively.

As far as their taxonomy is concerned, several entities are listed in the recently updated WHO classification, including nodal, extra-nodal and leukemic forms [3]. Of note, significant advances resulting from recent transcriptomic or genomic studies were incorporated in the current classification that may soon affect the clinical practice. Among nodal PTCLs in particular, an umbrella category designated 'nodal T-cell lymphomas of T-follicular helper (TFH) origin' has been introduced [4]. It includes AITL, follicular T-cell lymphoma and other nodal PTCL with a TFH phenotype, likely constituting

\section{KEYWORDS}

- classification - molecular biology

- new drugs • peripheral

T-cell lymphoma • stem cells transplantation $\bullet$ targeted therapy 
"In all instances, the main issue will certainly be to identify biomarkers

to select patients particularly sensitive to a specific drug." the most prevalent category in many countries $[4,5]$. These lymphomas show overlapping pathological and genetic features, especially recurrent mutations in epigenetic modifiers (TET2, DNMT3A and IDH2), in the RHOA GTPase and in T-cell receptor related genes, some of which could impact therapy [4,6-9]. Among ALCLs, besides the well-defined ALKpositive entity, ALK-negative ALCL is now a definite entity. However, ALK-negative ALCL probably encompasses genetically heterogeneous diseases, including the subsets with IRF4/ DUSP22 rearrangements and TP63 abnormalities, characterized by good and bad outcome, respectively $[4,10]$. Furthermore, a new rare provisional entity, 'breast implant-associated ALCL', occurring as either an effusion around the implant or as a mass adjacent to the breast implant, is now present $[3,4]$. PTCL/NOS still remains the most common entity and includes discrete subtypes characterized by diverse cellular counterparts [11]. In particular, the expression of the Th2 (GATA3) or Th1 (TBX21) associated master transcription factors, or of cytotoxic molecules seems to be associated to peculiar clinical behavior [12]. At the genetic level, PTCLs/ NOS are quite heterogeneous with different molecular targets being identified in the last few years [13]. Particularly, mutation-induced T-cell receptor activation and costimulatory signaling pathways (FYN, CD28, PLCG1, PI3K and CARD11 mutations; ITK-SYK, CTLA4-CD28 and CTLA4-ICOS gene fusions; DUSP22 and $V A V 1$ rearrangements) have emerged as an oncogenic mechanism in AITL, TFH-PTCL, PTCL/NOS, ALK-negative ALCL, cutaneous T-cell lymphomas and ATLL. Mutationinduced activation of the JAK-STAT pathway (JAK1, JAK3, STAT3, and STAT5B mutations) is a pathogenic mechanism common to several PTCL entities, including T-cell leukemias and extranodal PTCLs [14].

As far as PTCL first-line treatment is concerned, as mentioned, currently available conventional chemotherapy regimens have failed to significantly improve patients' survival in the last decades $[2,15]$. As a result, a clinical trial is still preferred for newly diagnosed PTCL patients in the NCCN guidelines [16]. Nonetheless, the addition of etoposide to CHOP (cyclophosphamide, doxorubicin, vincristine and prednisone) regimen (CHEOP) and the use of frontline autologous stem cell transplantation might have some favorable impact (NLG-T-01 trial; NCT00791947) [17].
The combination of CHOP with new agents that have different mechanisms of action and therapeutic targets was tried in clinical trials including the anti-CD52 monoclonal antibody (alemtuzumab), proteasome inhibitor (bortezomib), antivascular endothelial growth factor antibody (bevacizumab), denileukin diftitox and mTOR inhibitor (everolimus). Most studies reported the potential benefit of the addition of a new agent to the CHOP backbone, showing the improved response rate in newly diagnosed PTCL patients [18]. However, relatively short duration of response and the occurrence of significant toxicities, such as infection, limited the value of those combinations. Moreover, those studies were Phase II trials with relatively small numbers of patients. Thus, further studies with larger study population and more effective novel combination regimens are needed for PTCL patients; currently, Phase III trials adding other agents to the CHOP backbone are ongoing, such as the histone deacetylase inhibitor (HDACi), romidepsin (NCT01796002) and the anti-CD30 monoclonal antibody, brentuximab vedotin (BV) (NCT01777152). On the other hand, a few new agents have been then approved for clinical use in relapsed/refractory PTCLs, including praletexate, romidepsin, belinostat and BV. Pralatrexate is an antifolate chemotherapy with high affinity for the reduced folate carrier-1 receptor and folylpolyglutamate synthase. In Phase II trials, it induced a $29 \%$ overall response rate (ORR), including 12 complete remissions (CR) in relapsed/refractory PTCLs. The median OS and PFS were 14.5 and 3.5 months, respectively [19]. Of note, the toxicity of pralatrexate can be considerable. Oral mucositis can occur in spite of supplementation with folic acid and vitamin B12, immunosuppression and thrombocytopenia being also common $[18,19]$. Therefore, pralatrexate may be unsuitable for some patients, especially frail/elderly or those with relevant comorbidities $[18,19]$.

Romidepsin is a potent histone deacetylase inhibitor that alters gene transcription by interfering with the acetylation pattern of chromatin on histone lysine residues thus affecting protein function in cancerous cells. In the pivotal Phase II study, the ORR was 25\%, including $15 \%$ of confirmed/unconfirmed complete response (CR/CRu) [20]. The median PFS was 17 months; notably, some patients had long-term CR (>34 months) [18,20]. Side effects were similar to those of other HDACi, and mainly included hematological adverse effects such as anemia, 
thrombocytopenia and leukopenia [20].

Belinostat, is a multiple classes $(1,2,4)$ HDACi, with clinical efficacy quite similar to romidepsin. In fact, the ORR was 26\% (CR rate $11 \%$ ) while the median PFS and OS were 1.6 and 7.9 months, respectively. Sustained remissions ( $>3$ years) were observed in some patients as with romidepsin [18,21]. Remarkably, it was observed that belinostat might have particularly high efficacy in AITL patients (ORR 46\%) [18,21]. The toxicity profile was overall mild, suggesting a potential role in combination regimens as well as in frail patients with pre-existing thrombocytopenia or anemia $[18,21]$.

$\mathrm{BV}$ is an antibody-drug conjugate directed against CD30. This surface marker is typically expressed in Hodgkin lymphoma and ALCL. However, a significant percentage of other PTCLs and exceptionally nonhematopoietic neoplasms also expresses CD30. BV is composed by the anti-CD30 chimeric antibody cAC10 and its conjugate monomethyl auristatin E, a microtubule-disrupting agent. In systemic ALCL, the initially reported ORR was $86 \%$, with $57 \%$ of CR (median duration, 13.2 months), despite the high prevalence of primary refractory patients. Noteworthy, the 4-year OS was still 64\%, with a median PFS for the entire cohort of 20 months [22]. The most commonly observed toxicities were peripheral sensory neuropathy, pyrexia, nausea, constipation or diarrhea, fatigue, rash and neutropenia [18,22]. Grippingly, retreatment with BV of patients experiencing relapse after initial complete or partial response obtained with a prior BV therapy, is still highly effective in ALCL patients, with 63\% CR rate, 88\% ORR and a median PFS of 12.3 months [18]. Subsequent trials pointed toward the clinical activity of BV also in other PTCL subtypes, including PTCL/NOS, AITL as well as cutaneous lymphomas. Interestingly, it was observed that there was no direct correlation between CD30 expression and objective responses, clinical activity being noted even in cases with undetectable CD30 expression [18]. More sensitive tests, such as multispectral image analysis, may be more effective when immunohistochemistry fails in CD30 detection [18].

Additionally, a variety of novel drugs of different classes are currently under investigation in nodal PTCLs. These investigational agents include chemotherapeutic agents (bendamustine, forodesine, plitidepsin), Aurora kinase inhibitors (alisertib), HDAC (panobinostat), proteasome inhibitors (bortezomib), immunomodulatory drugs (lenalidomide), tyrosine kinase and PI3K inhibitors (crizotinib, duvelisib), denileukin diftitox, selinexor and monoclonal antibodies (alemtuzumab, mogamulizumab) [18]. In all instances, the main issue will certainly be to identify biomarkers to select patients particularly sensitive to a specific drug.

Finally, concerning allogeneic stem cell transplantation, although it has been shown to be potentially curative for approximately $40 \%$ of patients affected by relapsed/refractory PTCL, at present there is no evidence that allogeneic stem cell transplantation is superior to autologous stem cell transplantation for patients in first remission after induction therapy [23].

In conclusion, despite PTCL prognosis is still dismal, the recent recognition of cellular derivation and genetic background of these tumors, coupled with the availability of novel agents have probably opened a new era with new hopes for PTCL treatment.

\section{Financial \& competing interests disclosure}

The author has no relevant affliations or financial involvement with any organization or entity with a financial interest in or financial conflict with the subject matter or materials discussed in the manuscript. This includes employment, consultancies, honoraria, stock ownership or options, expert testimony, grants or patents received or pending, or royalties.

No writing assistance was utilized in the production of this manuscript.

\section{References}

1 Jaffe ES, Harris NL, Stein H, Campo E, Pileri SA, Swerdlow SH. Introduction and overview of the classification of the lymphoid neoplasms. In: WHO Classification of Tumors of Hematopoietic and Lymphoid Tissues. Swerdlow S, Campo E, Harris NL et al.
(Eds). IARC, Lyon, France, 158-166 (2008).

2 Bellei M, Sabattini E, Pesce EA et al. Pitfalls and major issues in the histologic diagnosis of peripheral T-cell lymphomas: results of the central review of 573 cases from the T-Cell Project, an international, cooperative study. Hematol. Oncol. doi:10.1002/ hon.2316 (2016) (Epub ahead of print).
2016 revision of the World Health Organization classification of lymphoid neoplasms. Blood 127(20), 2375-2390 (2016).

4 Gaulard P, De Leval L. Classification of T-cell lymphomas: where do we stand? Presented at: The 9th Annual T-Cell Lymphoma Forum. San Francisco, CA, USA, 26-28 January 2017.

5 Ahearne MJ, Allchin RL, Fox CP, Wagner 
SD. Follicular helper T-cells: expanding roles in T-cell lymphoma and targets for treatment. Br. J. Haematol. 166(3), 326-335 (2014).

6 Lemonnier F, Couronne L, Parrens M et al. Recurrent TET2 mutations in peripheral T-cell lymphomas correlate with TFH-like features and adverse clinical parameters. Blood 120 (7), 1466-1469 (2012).

7 Wang C, Mckeithan TW, Gong Q et al. IDH2R172 mutations define a unique subgroup of patients with angioimmunoblastic T-cell lymphoma. Blood 126(15), 1741-1752 (2015).

8 Palomero T, Couronne L, Khiabanian $\mathrm{H}$ et al. Recurrent mutations in epigenetic regulators, RHOA and FYN kinase in peripheral T cell lymphomas. Nat. Genet. 46(2), 166-170 (2014)

9 Vallois D, Dobay MP, Morin RD et al. Activating mutations in genes related to TCR signaling in angioimmunoblastic and other follicular helper T-cell-derived lymphomas. Blood 128(11), 1490-1502 (2016).

10 Parrilla Castellar ER, Jaffe ES, Said JW et al. ALK-negative anaplastic large cell lymphoma is a genetically heterogeneous disease with widely disparate clinical outcomes. Blood 124(9), 1473-1480 (2014).

11 Piccaluga PP, Agostinelli C, Tripodo C et al. Peripheral T-cell lymphoma classification: the matter of cellular derivation. Expert Rev.
Hematol. 4(4), 415-425 (2011).

12 Iqbal J, Wright G, Wang C et al. Gene expression signatures delineate biological and prognostic subgroups in peripheral T-cell lymphoma. Blood 123(19), 2915-2923 (2014).

13 Schatz JH, Horwitz SM, Teruya-Feldstein J et al. Targeted mutational profiling of peripheral T-cell lymphoma not otherwise specified highlights new mechanisms in a heterogeneous pathogenesis. Leukemia 29(1), 237-241 (2015).

14 De Leval L, Gaulard P. Molecular profiling to improve classification of T-cell lymphomas. Presented at: The 9th Annual T-Cell Lymphoma Forum. CA, USA, 26-28 January 2017.

15 Vose J, Armitage J, Weisenburger D. International peripheral T-cell and natural killer/T-cell lymphoma study: pathology findings and clinical outcomes. J. Clin. Oncol. 26(25), 4124-4130 (2008).

16 Horwitz S, Zelenetz A, Gordon L et al. NCCN Guidelines Insights: non-Hodgkin's lymphomas, version 3.2016. J. Natl Compr. Canc. Netw. 14(9), 13 (2016).

17 D'amore F, Relander T, Lauritzsen GF et al. Up-front autologous stem-cell transplantation in peripheral T-cell lymphoma: NLG-T-01. J. Clin. Oncol. 30(25), 3093-3099 (2012).

18 Zinzani PL, Bonthapally V, Huebner D, Lutes
R, Chi A, Pileri S. Panoptic clinical review of the current and future treatment of relapsed/ refractory T-cell lymphomas: peripheral T-cell lymphomas. Crit. Rev. Oncol. Hematol. 99, 214-227 (2016).

19 O'connor OA, Pro B, Pinter-Brown L et al. Pralatrexate in patients with relapsed or refractory peripheral T-cell lymphoma: results from the pivotal PROPEL study. J. Clin. Oncol. 29(9), 1182-1189 (2011).

20 Coiffier B, Pro B, Prince HM et al. Results from a pivotal, open-label, phase II study of romidepsin in relapsed or refractory peripheral T-cell lymphoma after prior systemic therapy. J. Clin. Oncol. 30(6), 631-636 (2012).

21 O'connor OA, Horwitz S, Masszi T et al. Belinostat in patients with relapsed or refractory peripheral T-Cell lymphoma: results of the pivotal Phase II BELIEF (CLN-19) study. J. Clin. Oncol. 33(23), 2492-2499 (2015).

22 Pro B, Advani R, Brice P et al. Brentuximab vedotin (SGN-35) in patients with relapsed or refractory systemic anaplastic large-cell lymphoma: results of a Phase II study. J. Clin. Oncol. 30(18), 2190-2196 (2012).

23 Perrone G, Farina L, Corradini P. Current state of art for transplantation paradigms in peripheral T-cell lymphomas. Expert Rev. Hematol. 6(4), 12 (2013). 